

\title{
SUMMARY OF IAU COLLOQUIUM 191: THE ENVIRONMENT AND EVOLUTION OF BINARY AND MULTIPLE STARS
}

\author{
Edward F. Guinan ${ }^{1}$ \\ RESUMEN
}

Se presenta un resumen del Coloquio 191 de la UAI "El Entorno y la Evolución de las Estrellas Binarias y Múltiples". Esta conferencia se celebró en Mérida, México, en febrero de 2003, y estuvo dedicada al Dr. Arcadio Poveda, por sus importantes contribuciones al campo de las estrellas binarias y múltiples y su dinámica. La conferencia se organizó para discutir los principales desarrollos que están ocurriendo en los campos de formación de binarias y múltiples, su evolución, su dinámica y el entorno en donde se forman y evolucionan.

\section{ABSTRACT}

A summary is given of the IAU Colloquium No 191 "The Environment and Evolution of Binary and Multiple Stars." This conference was held in Mérida, Mexico during February 2003 and dedicated to Dr. Arcadio Poveda for his major contributions to binary- and multiple-star systems and dynamics. The conference was organized to discuss the major developments that are occurring in the fields of binary- and multiple-star formation, evolution, dynamics, and the environments where they form and evolve.

\section{Key Words: BINARIES: GENERAL - GALAXY: OPEN CLUSTERS AND ASSOCIATIONS - STARS: FORMATION - STARS: EVOLUTION - TECHNIQUES: HIGH ANGULAR RESOLUTION}

\section{INTRODUCTION}

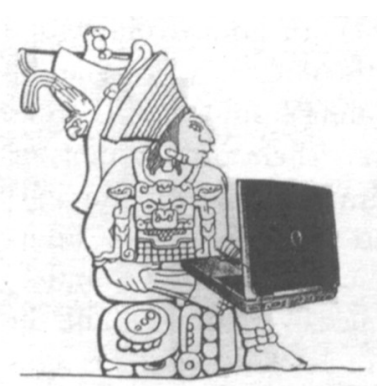

This was a very successful and interesting meeting, held in the beautiful city of Mérida, Mexico, capital city of the Yucatan State. The meeting was organized by Colin Scarfe and Christine Allen and held in honor of Dr. Arcadio Poveda who was born in Mérida. It is fitting that this conference was held in the heart of Maya land and near famous Mayan archeological sites, such as Uxmal and Chichén Itzá, because the Maya are known for their careful astronomical observations and the famous (and accurate) Mayan calendars. Also, because the conference includes $\mathrm{n}$-body interactions and collisions, it is fitting that the conference is located near the Chicxulub impact site which is associated with the K-T extinction some 65 Myr ago.

About 80 people attended the colloquium from over 20 different countries. The primary aims of the conference were the up-to-date exchange of information and ideas on the rapid advances and developments made over the last decade in the study of

\footnotetext{
${ }^{1}$ Villanova University, U.S.A.
}

both close and wide binaries and multiple star systems. It is noteworthy that much of the progress in these fields arises from vastly improved parallaxes for a large number of stars, made primarily from Hipparcos. Also important are high precision radial velocities (e.g., from CORAVEL and others), optical, infrared and radio interferometry, as well as direct, high resolution images from ground-based adaptiveoptics (AO) telescopes and from the Hubble Space Telescope. On the theoretical side, more powerful, high speed computers, as well as more realistic, sophisticated modeling and simulations of binary and multiple star interactions, have led to new insights into the formation, dynamical evolution and stability of binary and multiple star systems. This modeling, in fact, explains much (but not all) of what is now observed in terms of the distribution and frequencies of binary as well as hierarchical star systems in the field and in star clusters.

\section{OBSERVATIONAL PROPERTIES AND STATISTICS OF BINARY STARS IN THE FIELD}

Several papers were presented about the statistical properties of binary and multiple star systems. Andrei Tokovinin discussed the statistics of stellar systems with multiplicities of three and higher and studied the distributions of commonly measured orbital properties such as eccentricity, orbital inclina- 


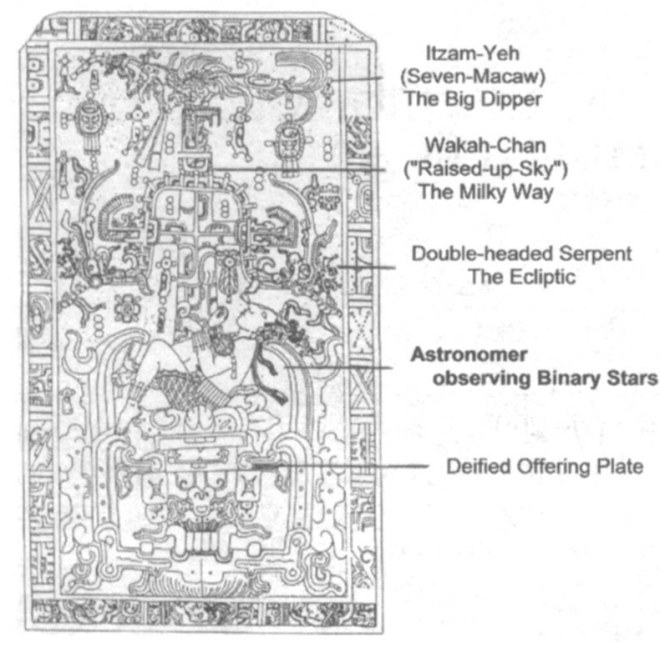

Fig. 1. The Wakah-Chan Tree and the Ecliptic Snake

tion, period, and mass ratio. This study of mainly nearby field stars indicates that multiple star systems (MSS) are frequent and represent 15-25 percent of all stars. It also appears that many (maybe all?) close binaries have companions and that about $1 / 3$ of visual binaries in the field are MSS. However, when a volume-limited sample of $\mathrm{d}<50 \mathrm{pc}$ is investigated, only 76 MSS are found, whereas nearly 10 times that amount are expected from solar neighbor studies. He asked "where are these expected MSS hiding?" From the sample he discussed the implications for star formation, cloud collapse, and the importance of Kozai cycles in star-star tidal interactions. He also questioned the long-term stability of many triple systems and pointed out some unusual multiple systems that defy explanation under current ideas of formation and evolution. In another interesting statistical study, Netzach Farbiash and Raphael Steinitz reported on the investigation of correlations between rotational spin velocities in a sample of over 1000 binary systems. They found significant correlations between the projected spins of the components of binaries. Checks against artificial binaries indicate that the observed correlations are real and that their results indicate that the spin axes of the binary components are preferentially parallel and oriented perpendicular to their orbital planes. This result is relevant to the various theories of formation and evolution of binary stars discussed at this conference. Further work is planned to determine the orbital periods of the sample stars.

Jean-Louis Halbwachs (with Udry and Arenou) reported on the analysis of a more limited sample of solar type field stars with spectral types of F7 - K.
Their study was based on CORAVEL radial velocities of field and open-cluster solar-type stars. The use of Hipparcos parallax data permitted an unbiased sample of stars to be studied. They detected nearly 90 spectroscopic binaries in the sample with $\mathrm{P}<10$ yrs. About 14 percent of the stars investigated are spectroscopic binaries (SBs); no significant difference in stellar properties was found between field stars and cluster members. This study indicated a bi-modal distribution in properties (two broad period peaks near $\sim 20$ days and 750 days), which perhaps suggests two different processes that generate close $(\mathrm{P}<10 \mathrm{yrs})$ binary systems. In a related paper, Anne Eggenberger (with Halbwachs, Udry and Mayor) discussed the extension of the original Duquennoy \& Mayor (1991) CORAVEL study by adding additional K-stars with Hipparcos parallaxes to the sample and extending the time base to over a decade. They presented initial results on the statistical properties of an unbiased sample of F7-K stars. This work focused on the long period systems. Their results indicated that binary stars are the dominant mode of star formation - that is "more stars are found in binary and multiple systems than not." They also found that twins (stars of equal mass) are common in the shorter-period binaries.

Hans Zinnecker, with co-authors Köhler and Jahreiss, presented the results of a study of visual binaries and multiple stars among Population II stars. The stars were selected from their proper motions and nearly 200 stars were investigated for duplicity and multiplicity using infrared interferometry, adaptive optics and wide field imaging. They found 31 binaries and 6 triples among this sample. This indicates a binary frequency of $15-25 \%$ which is similar to field stars but much lower than the frequency found in young clusters. As discussed in their paper, the study of old (halo/metal-poor binaries) is important because these systems were formed under very different conditions than were younger disk stars.

Helmut Abt and Daryl Willmarth presented the results of their ongoing radial-velocity investigation of nearly $300 \mathrm{~F} 7-\mathrm{K} 7$ stars. This study is being carried out primarily to search for very low-mass companions and to determine if there is a "brown-dwarf desert" among binary members. With an average precision of $100 \mathrm{~m} / \mathrm{s}$, their study can discover secondaries with masses down to about $0.01 \mathrm{M}_{\odot}$. The observations made so far indicate that over 20 percent of stars have significant radial-velocity variations. It will be interesting to see if this study finds the missing brown-dwarf secondaries.

The distributions of the orbital separations of 
double and multiple star systems were reviewed and evaluated by Arcadio Poveda and Christine Allen. They found that the distributions of the semi-major axes of these systems carry important information about the conditions and mechanisms of formation, as well as the dynamical evolution of the orbital properties of the stars over time as they encounter molecular clouds, spiral arms, massive stars etc. An important aspect of this research is the dissolution times of the wider systems. For the purpose of the study, the systems were segregated into different age groups (primarily based on space motions and metallicities), and orbital properties (i.e. separation) from various catalogs. In this paper, Poveda and Allen tried to make sense out of dissolution times of binary systems, frequency distributions of major axes, runaway stars, and binary star formation and destruction processes. One of the more interesting results of their study is that the distributions of the orbital separations of all age groups (from very young to old disk stars) seem to follow the classic 1925 Öpik distribution law - i.e.:

$$
\mathrm{f}(\mathrm{s}) \mathrm{ds} \sim \mathrm{ds} / \mathrm{s}
$$

in which " $\mathrm{s}$ " is the observed separation between components (see Öpik 1925; 1929). They also explained most runaway stars as resulting, not so much by ejections from binaries in which one component undergoes a supernova explosion, but more likely from ejections from close encounters that occur in more dense (possibly contracting) star forming regions. I am sure that Dr. Öpik would be happy that his distribution law apparently still holds true today even though why it does so is not fully explained.

In a related paper on binary star separations, Michael Sterzik addressed the vexing question "Are binary star separations related to their systemic masses?" He presented the results of various studies of field binaries with very low mass (dM8+ stars), low mass (dM2-dM5 stars) and binaries with solar mass primaries. Dynamical decay models predict an increase in multiplicity with increasing systemic mass chiefly because the more massive systems are more tightly bound and can survive longer. The frequency of field solar-type binaries is $\sim 57 \pm 10 \%$, while low-mass (dM-type) binaries have a frequency of $31-42 \%$ (depending on the particular study). However, very low-mass systems are much less common with a frequency of $10-15 \%$. Sterzik concluded that binary frequency distributions do appear to be dependent on their systemic masses.

After years of development, major new longbaseline interferometers are playing important roles in binary and multiple star research. Instruments like CHARA and the Navy Prototype Optical Interferometer (NPOI), along with others, are beginning to produce some very interesting results. An example of this potential was shown in the report by Christian Hummel of observations of the hierarchical triple system $\eta$ Vir with NPOI. In this study all of the components of this triple system were resolved and their relative positions were measured over time with a precision of \pm 0.07 mas. From the astrometric and spectroscopic data, the orbital and physical properties of the stars were determined for the first time, with some interesting astrophysical implications.

High-precision red spectroscopic observations carried out recently by Frank Fekel revealed an increased multiplicity in three well studied binary and triple systems, V815 Her (an SB1 binary), and HD 140122 and 77 Cyg (both known triple systems). Frank related a fascinating saga of luck, perseverance, a "midnight mistake" and the "unexpected expected results" in unraveling the secrets of these three star systems.

\section{NOMENCLATURE OF MULTIPLE STAR SYSTEMS}

In a special session at IAU Colloquium 191,

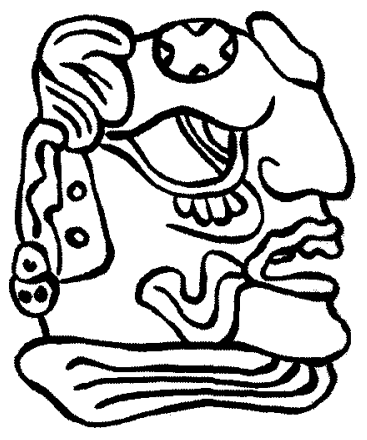
William Hartkopf and Brian Mason discussed the current status of an effort to address the growing confusion in double and multiple star nomenclature brought about by major advances in technology and reduction methods. New techniques that include interferometry (both long-baseline and speckle), adaptive optics $(\mathrm{AO})$, and high-precision spectroscopy have led to a rapid proliferation in the number of stars with identified companions. Also, the discovery of increasing numbers of new object classes - such as extra-solar planets and brown dwarfs as companions to single and double stars adds to this problem. For example, interferometry and $\mathrm{AO}$ techniques now permit measurement of the angular separation of many stars previously not known to have companions, and the resolution of greater numbers of spectroscopic binaries into astrometric systems. Moreover, during the next decade, 
astrometric and interferometric space missions such as the Space Interferometry Mission (SIM) and GAIA, as well as missions to search for extra-solar planets, such as COROT and Kepler, should lead to the discovery of hundreds to thousands of complicated multiple star/planet systems. The progress made on the adoption of a long-lasting, versatile nomenclature and designation scheme was discussed, and examples were presented in the context of the Washington Multiplicity Catalog (WMC). Every effort is being made toward getting this nomenclature system right, since, as Christian Hummel commented, "The future is longer than the past." A thorough discussion of this important topic, as well as additional information about the $\mathrm{WMC}$, can be found at the WMC website: ad.usno.navy.mil/wds/newwds.html.

At the conference, an amazing discovery was found for a possible Maya Multiplicity Star Codex. An example of the Maya system is given below for the multiple star system $\chi^{1}$ UMa.

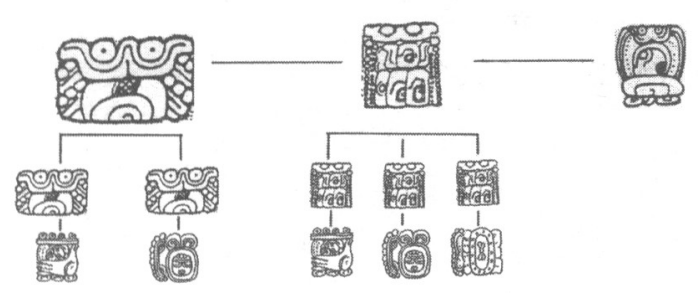

Fig. 2. Mayan Multiple Star Classification System as applied to $\chi^{1} \mathrm{UMa}$.

\section{OBSERVATIONAL RESULTS FOR VERY YOUNG BINARIES}

In a paper on multiplicity and outflow activity of newborn stars, Bo Reipurth discussed the strong observational evidence for multiplicity among the very youngest low mass stars. High-resolution images of a large number of Herbig-Haro (HH) objects display manifestations of significant outflows that include complicated bow shocks and jets. Spectacular images of several $\mathrm{HH}$ objects also show knots in the outflows and wiggles in the jets. As discussed by Reipurth, the $\mathrm{HH}$ flows represent fossil records of the birth of binary systems. Some of the observed periodic variations shown in the images are believed to be caused by multiplicity effects. The observed periodic outflows can arise as a binary companion approaches the periastron of its highly eccentric orbit. Simulations suggest that a very fast dynamic

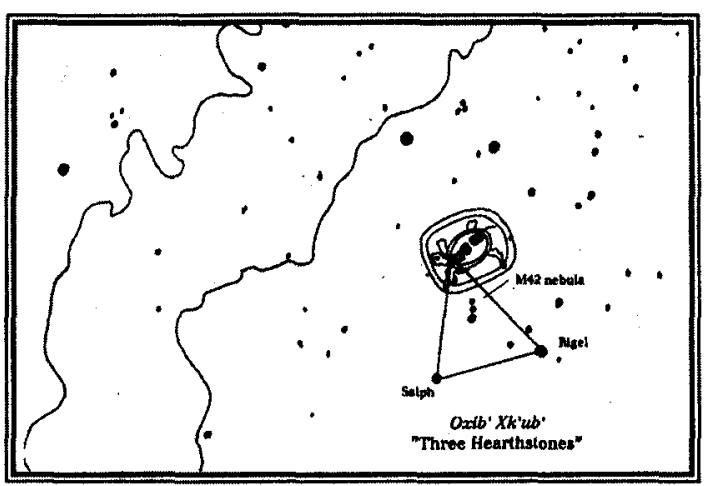

Fig. 3. Maya star map of the Orion region. The Maya constellation "Three Hearthstones" is shown. The Maya depicted Orion's belt as a turtle. The Orion Nebula (M42) and Trapezium cluster are shown.

evolution of the young binary system is occurring and that the orbits are often unstable and decay over time scales of $\leq 10^{5}$ years. Studies of $\mathrm{HH}$ objects indicate that most ( $\sim 80$ percent - if not all) giant $\mathrm{HH}$ objects are binary or triple systems. Understanding the formation and evolution of these objects is important because, as Bo Reipurth said, "If we do not understand how stars form, we cannot know how galaxies form."

In the paper, "Orbital Motions in Binary Protostellar Systems," Luis Felipe Rodriguez discussed high spatial resolution ( $\sim 100$ mas) Very Large Array (VLA) observations of several low-mass $(\sim 1$ $2 \mathrm{M}_{\odot}$ ) young-stellar-object (YSO) proto-binary star systems in the Taurus and $\rho$ Ophiuchi star-forming regions. The large luminosities observed for these YSOs support the idea that young protostars derive most of their luminosity from accretion processes and not from nuclear reactions. The rewards of carrying out systematic long-term observations were clearly demonstrated in this paper. By combining VLA observations carried out for nearly 20 years, longterm orbital motions have been detected for some of the targets. For example, in the case of the famous YSO prototype, T Tauri, VLA observations of its southern component, T Tau-S, reveal what appears to be orbital motion (defined by a partial astrometric ellipse) between 1983 and 1998. However, observations made after 1998 do not appear to fit the preliminary astrometric orbit and indicate that the star is not following the expected orbital behavior. Rodriguez suggested that perhaps the orbit was disrupted sometime after 1998. - maybe an ejection occurred? However, recent optical and near-IR astrometry of T Tauri-S using the NPOI seems to contradict 
this suggestion - see Hummel et al. (2004 - in press). The planned additional observations should clarify this interesting situation. Rodriguez and collaborators plan more radio observations with the VLA and Very Long Baseline Interferometer (VLBI). In the next several years, improved high spatial resolution observations of YSOs with the Expanded Very Large Array (EVLA), Atacama Large Millimeter Array (ALMA), and the Square Kilometer Array (SKA) should produce some very exciting results and provide important constraints on the early phases of stellar evolution.

Guillem Anglada presented a paper on VLA observations of disks and jets of selected young close binaries. High angular resolution observations now available at radio wavelengths make it possible to resolve the binary components and in some cases detect small orbital motions. Observations of SVS13, NGC 1333-VLA2, and L723-VLA2 indicate that these radio sources are very young, close (25-75 AU separation) binary systems. Moreover, there is strong evidence that NGC 1333-VLA2 is a young multiple star system with a separation of $\sim 25 \mathrm{AU}$ in which one component dominates the flux at $3.6 \mathrm{~cm}$ while the other component dominates at $7 \mathrm{~cm}$. These results suggest that the development of disks and jets can proceed in different ways for each component a binary system. Observations of higher angular resolution, and the improved signal-to-noise expected in the near future, of these and additional young radio sources, will be important to help understand the formation and properties of the disks and jets in very young star systems.

No meeting on binary stars would be complete without a paper about the "Mother of all binaries," $\eta$ Carinae. This star came to the attention of southern observers when it first dramatically brightened (known as the "Great" eruption) during the 1840s. Since that time, $\eta$ Car has been extensively studied and has been identified as (one of) the most massive binary stars in our galaxy. Yousaf Butt reported on X-ray observations of $\eta$ Car made with ROSAT, ASCA, RXTE, Beppo-SAX, and CHANDRA as well as coordinated near-IR and radio observations. Spectroscopic and X-ray observations secured over the last decade reveal the presence of a period of $P=5.52$ years. The variations in the observed X-ray emissions are believed to arise from collisions of the binary star components with strong stellar winds. X-ray eclipses are observed every 5.52 years during periastron passages and are believed to arise when the line-of-sight to the shocked gas is partially obscured or eclipsed. He discussed the plans

to observe $\eta$ Car during June 2003, when the Xray dimming is expected to occur during the putative periastron passage. They include coordinated high energy observations. ( $\gamma$-ray) made with INTEGRAL and X-ray observations made with CHANDRA. These observations should provide an important test of the still-disputed binary hypothesis of $\eta$ Car. This popular binary model predicts an enhancement in the $\gamma$-ray flux shortly after periastron passage near the time of the X-ray "eclipse." Consultation with IAU Circulars indicates that the X-ray eclipse occurred as expected during June 2003 (see IAU 8137/38). The results from INTEGRAL have yet to be reported at the time of writing.

\section{OBSERVATIONAL RESULTS FOR BINARY STARS IN CLUSTERS}

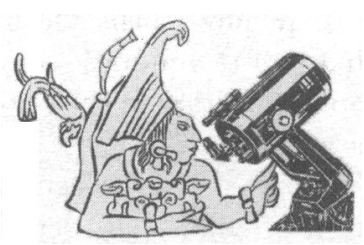

Fig. 4: Searching for companions to binary star systems. $\mathrm{km} / \mathrm{s}$ accuracy can now be obtained for solar type stars in nearby $(\mathrm{d}<300 \mathrm{pc})$ clusters. Moreover, the availability of multi-object fiber feeds permits simultaneous observations of up to hundreds of stars each night. This has lead to the identification of nearly all spectroscopic binaries with periods less than about several years in nearby open clusters. Several papers were presented that focus on the observations of binaries in clusters while two papers dealt with the relatively new field of binaries in nearby galaxies.

In the paper "Binaries in Open Clusters: Orbital Parameters and Stellar Astrophysics," Bob Mathieu discussed the results of his detailed study of the solar age $(\tau \sim 4.5 \mathrm{Gyr})$ open cluster M67. Many binary and multiple star systems have been detected in this rich cluster. The frequency of spectroscopic binaries in $\mathrm{M} 67$ is $19 \pm 4 \%$. This value is comparable to field spectroscopic binaries but is probably also an underestimate of the number of binary systems in M67 because the long period binaries are underrepresented. Precise photometry of the cluster indicates that 'photometric' binaries (i.e., stars that lie $\sim 0.5$ 0.75 mag above the cluster main sequence) have a frequency of $\sim 38 \%$. When selection and sampling effects are accounted for, the expected number of 
binaries in M67 is quite high, leading Mathieu to remark that "M67 should not be considered a cluster of stars but a cluster of binaries." In his "Day at the Zoo," Mathieu briefly discussed the properties"of interesting and rare binaries in M67. These include numerous blue stragglers (seven of these are SBs with known orbits), an eclipsing binary (S986) located at the cluster turn-off, several coronal X-ray binaries (four W UMa systems) and some stars that may have evolved through the common-envelope stage of binary-star evolution. He discussed the presence of several unusual binaries in terms of binary-evolution theory (binary mass-transfer and loss) and the possible effects of collisions and encounters with other cluster members.

Rainer Köhler and an international team of investigators tried to answer the "age-old question" of what causes the low binary frequency in the Orion Nebula Cluster (ONC)? Using AO techniques, they studied the multiplicity of about 220 stars in the outskirts of the young ONC. The stars selected for study are located about 0.7 to $2 \mathrm{pc}$ from the cluster center - the Trapezium Cluster. From previous (less sensitive) studies the effectively zero-age ONC has been found to be populated by a much lower number of binaries and multiple stars than young star forming $\mathrm{T}$-associations. The major aim of this study was to search for the cause(s) of the apparent frequencies of solar and low mass binary and multiple star systems in this and other young clusters. The outer regions of the ONC were selected for study because these regions have fewer stars, and binaries and multiple star systems are less likely to be disrupted from tidal (dynamical) interactions. The results of this interesting study show that the multiplicity fraction of low(solar)-mass stars is low (about 5-8\%), and does not depend on the distance from the cluster core. However, the frequency of high mass multiple (OB) stars is higher in the inner regions of the ONC (about $30 \%$ ). The frequency appears to decrease from the center to the outskirts of the cluster.

Several more related papers were presented. Gene Milone presented the initial results of an analysis of the photometric light curves for the eclipsing and ellipsoidal binaries discovered in the globular cluster 47 Tuc with the Hubble Space Telescope by Ronald Gilliland and collaborators. So far these faint cluster binaries do not have radial-velocity curves, which severely limits what can be determined. $\mathrm{He}$ discussed an iterative method of determining the physical properties of these close binaries based on the light-curve analysis (using a modified version of the Wilson-Devinney analysis program) and theoret- ical isochrones from stellar-evolution codes. Because the age, distance, $[\mathrm{Fe} / \mathrm{H}]$, colors, and reddening of 47 Tuc members are well known, it is possible to use the Mass-Luminosity relation and color- $\mathrm{T}_{\text {eff }}$ relations in estimating the physical and orbital parameters of the stars. This method shows great promise and should also be useful in determining the physical properties of the thousands of eclipsing systems discovered over the last decade in the Magellanic Clouds and M31 from microlensing programs such as MACHO and OGLE.

In a related paper Christine Allen discussed the presence of young stars found far from the galactic plane. Two of the best studied cases of "runaway stars" are AE Aur and $\mu \mathrm{Col}$. As suggested decades ago by Allen and Poveda, the best explanation for finding these young stars where they do not belong is from interactions in the core of young clusters and subsequent ejections. In the same session Lex Kaper discussed the importance of high-mass $\mathrm{x}$-ray binaries to the understanding of massive star formation and evolution. He reported on coordinated x-ray and Hipparcos observations. He discussed the "Dutch Compromise" in which it appears likely that normal type II supernovae result in neutron stars while black holes result from more massive $\gamma$-ray bursters.

Mattias Eriksson and Hans Veenhuizen reported on the investigation of Fe II fluorescence by photoexcitation from accidental resonance (PAR) in about 30 symbiotic variable stars observed in the UV by IUE. PAR-mechanism-pumped Fe II line emission channels were discovered in 8 systems, including the well studied systems RR Tel, AG Peg, V1016 Cyg, and AG Dra. They discussed the information that can be gained about plasma physics and dynamics from the study of the PAR Fe II fluorescent lines in symbiotic stars with different orbital and stellar properties.

A short report on the initial results from coordinated photometry of the well known cataclysmic binary IR Gem was presented by Kam-Ching Leung on behalf of an international team lead by Li Zongyun. The observations took advantage of the longitudinal separation of $160^{\circ}$ between Nanjing and Nebraska to study various periodicities apparent in the light variations. The photometry of this 98.5 -min. binary showed complicated behavior during quiescence that is as interesting as that found during its outburst, as well as positive and negative superhump states. However, the complex light variations uncovered for this close binary are far from being understood. 


\section{ECLIPSING BINARIES IN THE GALACTIC BULGE AND NEARBY GALAXIES}

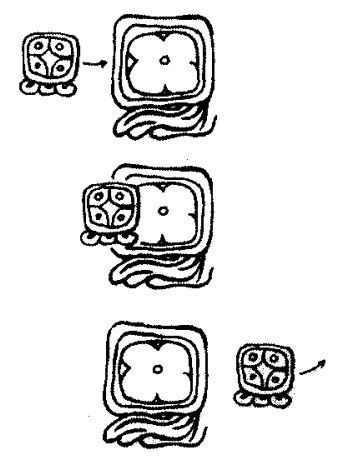

Fig. 5: Maya representation of an eclipsing binary star. thousands of additional eclipsing (and pulsating "single-star") variables are expected to be discovered in the near future from space missions such as the COROT, Eddington, Kepler, and GAIA missions. In her paper she discussed mining these data sets to understand the formation and evolution of close binaries - in particular for identifying the "missing links" of binary stellar evolution. She stressed the need to carry out the analysis of the light curves of large numbers of stars in an automatic way.

Glenn Bayne discussed photometry of eclipsing binaries in the Small and Large Magellanic Clouds being carried out as part of the microlensing observations in astrophysics program at Mount John University Observatory, New Zealand. Light curves for over one hundred eclipsing binaries have been obtained so far. In this talk he discussed the determination of photometric and orbital properties for two interesting systems. In a related paper, Guinan, Ribas, and Fitzpatrick discussed the progress of an ongoing HST and ground-based program of observing eclipsing binaries in the Local Group galaxies. This study is being made to determine accurate distances and the physical properties of the stars as well as probing the structure and evolution of their host galaxies. The analysis of the light and double-line radialvelocity curves yields stellar masses and radii; while the temperatures and interstellar extinction are measured from the HST 115-850 nm spectrophotometry. Independent measures of three eclipsing binaries located in the LMC bar region yield a distance of $\mathrm{d}=$ $48.3 \pm 1.6 \mathrm{kpc}$. The plans for determining the distance to the Andromeda galaxy (M31) using 19th20 th mag eclipsing binaries were also discussed. A review of this subject has been published recently

(Guinan 2004).

\section{THEORETICAL APPROACHES TO MULTIPLE SYSTEMS AND THEIR FORMATION}

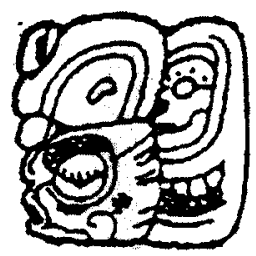

Fig. 6: Maya glyph indicating "captured" star. This could be relevant to several papers at the conference that focus on stellar mergers.
Mauri Valtonen discussed methods and solutions of the classical three-body problem. He pointed out that the solution of the threebody problem is important because three body processes play important roles in the dynamic evolution of many star clusters. Because modeling the exact 11-D solution of three-body orbits "could require computer runs near the age of the universe," he discussed solutions from 3-body perturbation theory and 3-body numerical methods. The solutions turn out to be either statistical or deterministic in nature, depending on the initial conditions. He applied these calculations to the observed properties of binary and multiple systems with some very interesting results.

In a related paper, Rosemary Mardling reported on a new formalism for studying three-body interactions. This new formalism permits the determination of the short-term and long-term stability of any triple-body configuration, along with determinations of energy and angular-momentum transfers during scattering and capture-process events. The procedure also gives the prediction for the final outcome of an unstable triple system and the study of resonance-capture processes. Some of the results for the effects of a third body on the orbits of close pairs were nicely illustrated with superb animations. For example, if the orbital eccentricity is large, the tertiary body can gain sufficient angular energy to be summarily ejected. In some cases this could explain "runaway stars." Interesting analogies in this treatment have been made to quantum mechanics. In some cases resonances can develop and the orbits of the third body and closer binary "talk to each other." Also, new criteria for the stability of three and " $n$ " body orbits have resulted from this work.

In another paper on the restricted three body problem, Ing-Guey Jiang presented new results that have interesting applications to the dynamics and stabilities of binary and multiple star systems, planetary systems, and even binary systems with black 
hole components. Examples of orbital interactions with different third-body masses and external forces were explored and the results discussed. He found that both the equilibrium points and solution curves can become quite different from those of the classical isolated three-body problem.

Before taking a hiking trip to traverse the high Andes, Sverre Aarseth presented a paper on the formation and evolution of hierarchical star systems. In that paper he gave an expert guided tour of the "hierarchical stellar zoo." His calculations (using realistic N-body simulations appropriate for more dense star forming regions) show that most compact triple systems are formed from binary-binary collisions. Triple systems tend to build up over time scales of 2-3 Gyr. Also, he finds that many stable (long-lived) quadruples and higher-order multiples can be expected. The higher orders of multiplicity can have various and diverse origins. Over time, external interactions can also cause an increase in the orbital eccentricity that can lead to instability of the system. Under some conditions, systems can be disrupted by a slingshot mechanism. Sometimes these reactions are sufficiently energetic to eject the stars with high velocity, thus resulting in "runaway stars." He discussed the expected properties of hierarchical systems such as quadruples, quintuples and sextuples. He made an interesting analogy between binary (and higher-order) systems, and molecules.

Tomoyuki Hanawa presented the results of 2-D numerical simulations of the accretion of gas from circumbinary disks to the primary and secondary stars of proto-binary systems. The somewhat surprising result of this study is that the primary (the more massive component) accretes more than its lower-mass companion. This seems to occur under various initial binary-system characteristics, and with different accretion conditions and orientations. In these simulations the accretion occurs onto the primary either through the L2 and L1 points, or through the L3 again (with the same result) onto the primary star. According to these simulations, the primary star's mass increases so that the mass ratio $\mathrm{M}_{s} / \mathrm{M}_{p}$ decreases with time. These simulations appear to be consistent with recent observations that the primaries in young binary systems have more massive accretion disks than the secondary stars.

Masahiro Machida discussed the application of three-dimensional MHD nested-grid simulations to study binary-star formation from a rotating magnetized molecular cloud. The results of these simulations indicate that the collapse of the magnetized gas depends on the amplitude of the non-axisymmetric

perturbations, the rotation speed, and strength of the magnetic field. The end result is the collapse of the gas and the formation of an opaque core. Depending on initial conditions, the resulting cores can have a wide variety of shapes that include bars, rings, disks and small spheres. Binary and multiple star systems can form from the bar, disk and ring models, while single stars form from the small spheres. These simulations could be scaled to study the formation and evolution of galaxies.

\section{ASTROPHYSICAL PROCESSES OF MULTIPLE STARS IN CLUSTERS}

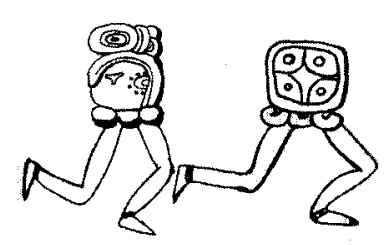

Fig. 7: Mayan glyphs representing runaway stars!
Matthew Bate discussed the results of new 3-D calculations. $\mathrm{He}$ studied the effects that star formation and evolution in dense star clusters have on the properties of binary and multiple stars. He employed a state of the art "Next Generation" 3-D Hydrodynamics code that yielded the first fully resolved calculations of fragmentation as small as $\sim 1 \mathrm{AU}$. He ran the simulations by varying the initial conditions of the cluster (changing mainly the initial density). The effects of accretion, interactions with circumbinary and circum-multiplesystem disks, dynamical interactions, and the initial density of the stars within the star-forming region were all studied. Fascinating animations of the fragmentation, accretion, and capture processes taking place over a 200,000 year time-scale were presented. About 70 percent of the stars in the cluster are expected to be binary and multiple star systems (in good agreement with observations for young star clusters). An interesting result of these studies is that the simulations initially produce nearly equal numbers of stars and brown dwarfs, but that the brown dwarfs result primarily from "arrested development", being ejected from the cluster before they can accrete enough matter to become stars. Close and wide binaries are formed as well as a variety of wide multiple-star systems. These simulations do not predict brown dwarfs as members of close solarmass pairs.

In a related study Cathie Clarke discussed the results of simulations of the formation (and disruption) of binary and multiple star systems in cluster environments. These simulations are different from the work done previously in that they model both the disruption processes produced from dynamical 
interactions and also the augmentation of the star masses from competitive accretion. As in the results of Bate, low-mass objects (like low-mass $\mathrm{dM}$ stars and brown dwarfs) appear to exist as a result of their early ejection, before they acquire sufficient gas to become stars. Thus only a few brown dwarfs are expected to be found in the cluster as single stars or members of close binary systems. Also, the modeling predicts that a significant number of brown dwarfs (and low mass late dM stars) could be hierarchical outliers of binary systems. It also has been found that binaries originating in the denser parts of the star-forming region (molecular cloud) have a higher gas supply and get a head start over the other stars. This advantage may result in higher-mass systems.

Christine Allen, with Poveda and HernándezAlcántara, revisited their old, young friend the Trapezium cluster. The chief aim of the new study was to examine the stability of a number of wide binary and multiple star systems in this very young cluster. In an earlier study, Allen and Poveda had found evidence that some wide multiple stars in this cluster are dynamically unstable. These systems should evolve by ejecting one or more components either into hyperbolic trajectories, or into loosely bound wide orbits. In the latter case these rapidly evolving systems are transformed into hierarchical configurations. This study was based on astrometry of the binary and multiple star systems dating back to 1830 . By carefully combining the older (less precise) data sets with more recent, more accurate, measures from the WDS Catalog of Observations, the authors found that most of the systems studied did not show significant motions. However, a few systems (e.g. ADS 2843 and ADS 13374) show large transverse motions that might indicate that they are escaping from the cluster. Overall, this study now uses about 170 years of observations and it lends support to the concept that the Trapezium binary and multiple stars are essentially unstable systems with lifetimes of a few million years. This work also confirmed the previous result of Allen and Poveda that there is no observational evidence for systematic expansion, or contraction, of the orbits of any of the systems. This study is an excellent illustration of the importance of historic data sets.

\section{VERY LOW MASS COMPANIONS (RED DWARFS \& BROWN DWARFS AND PLANETARY COMPANIONS)}

Stephane Udry, with colleagues Anne Eggenberger and Michel Mayor, discussed the detection and characteristics of extra-solar planets (ESPs) in

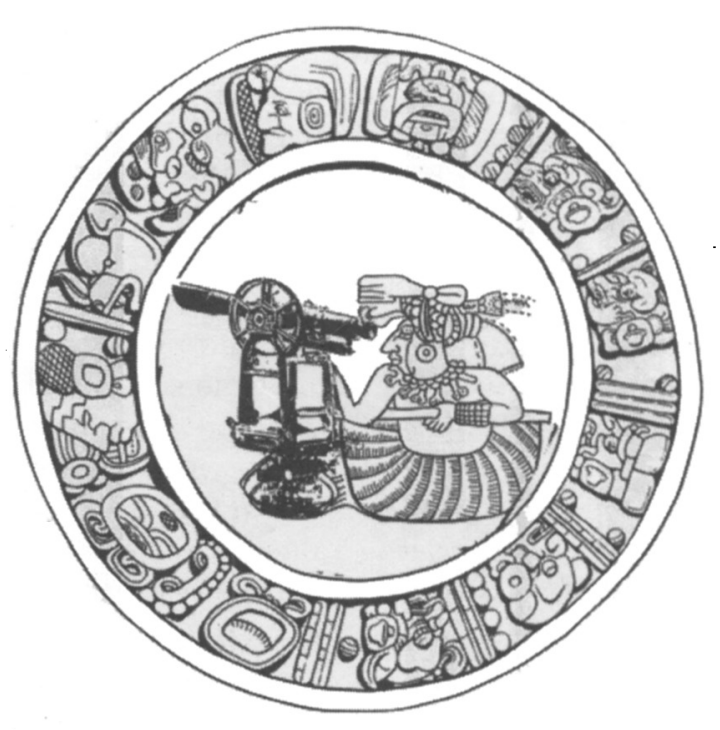

Fig. 8. Mayan astrometrist (possible ancestor to Arcadio Poveda?) shown inside Maya calendar.

multiple star systems. At the present time, there are $\sim 120$ known ESPs. Most of these orbit single mainsequence stars. Several stars (e.g. $v$ And and $\tau$ Boo) have two or more giant planets orbiting them. Surprisingly, a significant number of giant ESPs have highly eccentric orbits. This may arise from interactions, or a different manner of formation and evolution than binary stars. However, in their paper, the investigators primarily focused on searching for brown dwarfs and ESPs in binary systems (both spectroscopic and visual). About 12 ESPs have been found in binary systems. They confirmed the apparent paucity of brown-dwarf companions to stars, the so-called "brown-dwarf desert." So far ESPs have been found in two visual star systems, $16 \mathrm{Cyg}$ B and HD 80606. These stars are wide double-star systems and a planet would be stable orbiting close to one of the stars - as is the case. The investigators discussed their radial-velocity program of specifically searching for short period planets (and brown dwarfs) in singleline spectroscopic binaries (SB1s). They reported that none have been detected so far. Care must be taken in measuring the radial velocities of SB1 systems because many with cool-star components are expected to be magnetically active, having starspots and plages. These possible surface inhomogeneities could corrupt the measured RVs, leading possibly to spurious results or excessive scatter in the measures.

Ing-Guey Jiang and collaborators have examined the stabilities of Kuiper-belt-like structures around stars. This investigation was stimulated by the re- 
cent discovery of a dust ring around the young active early $\mathrm{K} 2 \mathrm{~V}$ star, $\epsilon$ Eridani: This disk or ring could be a young analog to a Kuiper-belt structure. In our solar system dozens of Kuiper-belt objects, mostly orbiting outside the orbit of Pluto, have been found so far. Jiang's investigation focused on the long-term stability of belts of that kind around stars with known planets. The results indicate that most extra-solar planetary systems can have Kuiper-beltlike populations at their outer regions. However, they report that Kuiper-belt structures are unstable in the presence of a massive planet with a highly eccentric orbit $(\mathrm{e}<0.8)$. The belt width also appears to increase with increasing values of the orbital eccentricities of massive planets in the system.

Rudolf Dvorak reviewed the status of observations and the theory of planets as members of binary star systems. So far, there is evidence of 7 planetary systems in binary stars - six are solitary planets and, in one system ( 55 Cancri), there are (at least) three planets. Planets can be stable in two configurations around binaries. In 'P'-type systems, a planetary orbit is stable far away from a close binary pair, while in 'S'-type systems, the planet orbits close to one of the stellar components of the wide binary pair (e.g. 16 Cyg AB). As an example, the author discussed the case of the $\gamma$ Cep system, in which a planet has been found in an eccentric orbit $(e=0.21)$ at 2.1 $\mathrm{AU}$ from one of the stellar components of this SB2 binary $(\mathrm{P}=70$ years; $\mathrm{a}=21 \mathrm{AU})$. The dependence of planetary-orbit stabilities on mass ratios, periods, inclinations, and separations was discussed, and interesting results were presented.

Thierry Forveille, in a paper presented by Stephane Udry, reported on the study of binary (and multiple star) characteristics of a volume-limited sample of about 100 low-mass dM stars. The characteristics of dM binary systems provide important diagnostics of star-formation processes, and the importance of gravitational interactions with other stars on these weakly bound low-mass systems over the age of the Galaxy. The multiplicity parameters (distribution of binaries, mass ratios, semi-major axes, eccentricities etc.) of this sample were determined and compared to a similar sample of solar-mass stars with some interesting results.

Ing-Guey Jiang discussed the formation of brown dwarfs. He discussed the current knowledge about the number and distributions of brown dwarfs in the field and in young star clusters, as well as their memberships in close and wide binaries. To explain their observed properties and distributions (especially the apparent rarity of brown dwarfs as members of bi- nary systems containing main-sequence stars), he proposed that the majority of brown dwarfs most likely form through secondary fragmentation processes. They are scattered, or removed, to large distances from the star-forming (gas-rich) region where they cannot grow into stars. Thus brown dwarfs result from a form of arrested development in which they are "thrown from their cradles early in life."

\section{NEW INSTRUMENTS, TECHNIQUES, AND PROGRAMS}

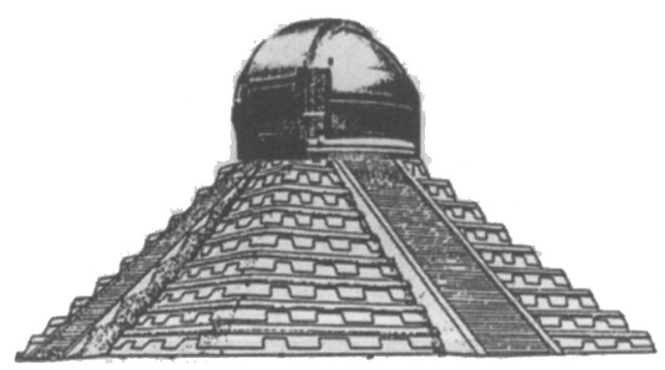

Fig. 9. Proposed observatory at Chichén Itzá.

Salvador Cuevas and his colleagues reported on an AO system being developed for the $2.1 \mathrm{~m}$ SPM UNAM telescope being built in the Baja. This AO system is named "GUIELOA," which, in the Zapotec language, appropriately means "Our Eyes." The site selected for the telescope has excellent average "seeing". The planned AO system covers the near-IR $(\mathrm{I}, \mathrm{J}, \mathrm{H}, \mathrm{K})$ and is expected to have very good angular resolution and fast read-out rates. Also, the system will have coronagraphic capabilities for investigating stellar disks and jets. Some of the planned applications of "GUIELOA" include the studies of binary and multiple star systems, as well as disks and jets of YSOs. The telescope and its instrumentation are expected to be operational by 2005 and should produce many new interesting results.

At this conference the detection of low mass companions (brown dwarfs and extra-solar planets) using high-precision radial-velocity spectroscopy and astrometry were discussed. Guinan, Ribas, and Arenou presented the results from an overlooked method of discovering low mass companions that uses the light-travel-time effect (LTTE). A tertiary body orbiting an eclipsing binary produces periodic variations in the observed arrival times of the eclipses. This occurs as the distance of the eclipsing binary varies as a result of its orbital motion around the barycenter of the triple system. The magnitude of 
the LTTE depends primarily on the mass of the tertiary body relative to the eclipsing pair, the orbital period of the tertiary system (i.e. the semi-major axis), the orbital inclination, and the eccentricity of the third body. Scores of companions to eclipsing binaries have been discovered using the LTTE, while Guinan and collaborators have discovered a probable brown-dwarf (Mass $\simeq 0.04 \mathrm{M}_{\odot}$ ) companion to the Hyades eclipsing ( $\mathrm{dK}+$ white dwarf) binary V471 Tauri. They reported on how high precision photometry and astrometry can be used as a method to detect low-mass stars and substellar objects in selected low-mass, uncomplicated, eclipsing binaries. In some cases the same method can also be applied to certain types of pulsating stars.

Andrea Richichi discussed long-baseline interferometry (LBI) and lunar-occultation (LO) methods for discovering and studying chiefly close binary systems. He discussed the statistical properties of the binary systems appearing in the CHARA Catalog (see Taylor et al. 2003) and those in the CDS. The LBI method is biased toward detecting binaries in which the magnitude difference of the components is $<3$ mag. The LO method has greater dynamic range with $\Delta \mathrm{m} \sim 4 \mathrm{mag}$ being possible. The advantages and disadvantages of both methods were discussed. The LO method is simple and straightforward, inexpensive, fast (takes $<1 \mathrm{sec}$ of observing time), yields angular separations of $\sim 1$ mas, and can detect components whose magnitude differences are up to 5 mag. However, the LO method is 1-D only, serendipitous (fewer than $10 \%$ of stars are occulted by the Moon), and has a fixed time series. The LBI method can select the targets from over the entire sky, and repetitive observations and angular resolutions of $<1$ mas are possible. However, the LBI method is expensive, requiring multi-telescopes and careful calibrations, the analysis is complicated, and the dynamic range is limited to $<3 \mathrm{mag}$. Also, the capabilities of ESO's Very Large Telescope Interferometer (VLTI - four $\times 8.2 \mathrm{~m}$ telescopes, baselines $=$ $130 \mathrm{~m}$ ) were discussed. The VLTI was referred to as "a door to the future of interferometry," and that is certainly true! Very exciting results on binary and multiple star systems are expected. Angular diameter measurements of the component stars are possible in many cases.

The GAIA Mission is planned to carry out microarcsecond astrometry of most stars brighter than $\sim 19$ th mag over a five-year mission. High-precision multi-band photometry, and low-dispersion spectroscopy, will be secured with GAIA over the expected lifetime of the mission. The targeted launch

date for GAIA is currently estimated for about 2013. GAIA is expected to discover several million new eclipsing binaries and provide useful light and radial velocity curves, as well as parallaxes for over 50,000 systems! In anticipation of this enormous and valuable data base, Tomaz Zwitter and Ulisse Munari are studying a small sample of nearby eclipsing binaries as a test bed for scripted automatic solutions of light-curve and radial-velocity data sets. These experimental datasets are similar to those expected to be delivered by GAIA. Test solutions are being made using a modified version of the Wilson-Devinney program. The initial results of this study are very encouraging.

\section{CONCLUSIONS AND CLOSING REMARKS}

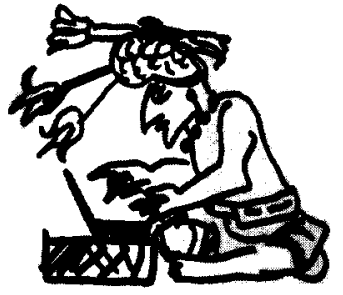

Fig. 10: Maya astronomer making last minute changes to his talk. matter. Unfortunately, there is no space to discuss them here. The poster papers include results from photometric and spectroscopic studies of individual objects, as well as present the results of surveys, and statistical and theoretical studies. They also cover a wide variety of objects from primordial stars to halo objects, as well as black holes. The reader should not overlook these many interesting papers.

The oral and poster papers presented here clearly demonstrate the amazing progress made over the last decade in the fields of binary and multiple stars and the effects of, and/or on, the environments in which these stars form and evolve. As discussed, new or improved instruments and techniques, as well as sophisticated modeling and improved theory, have brought about a renaissance in this once-neglected field. A colleague attending the colloquium, who is primarily known for his contributions to close binaries (mostly eclipsing binaries), confessed that, after attending the colloquium, maybe he didn't choose the right field of research.

Even though the progress made over the last decade has been great, many more exciting (and probably surprising) discoveries are expected dur- 
ing the next decade from new instruments and techniques, as well as advances in theory and modeling. During the next decade ground-based, radio, and optical facilities like ALMA, ELVA, SKA, and VLTI will be available. These, also with space-based missions such as Space Interferometry Mission (SIM), Spitzer Space Telescope (SST), James Webb Space Telescope (JWST), and later GAIA, along with others, should greatly expand our horizons and understanding of binary and multiple stars and will probably answer some questions raised here. However, they will most likely generate many new problems and questions.

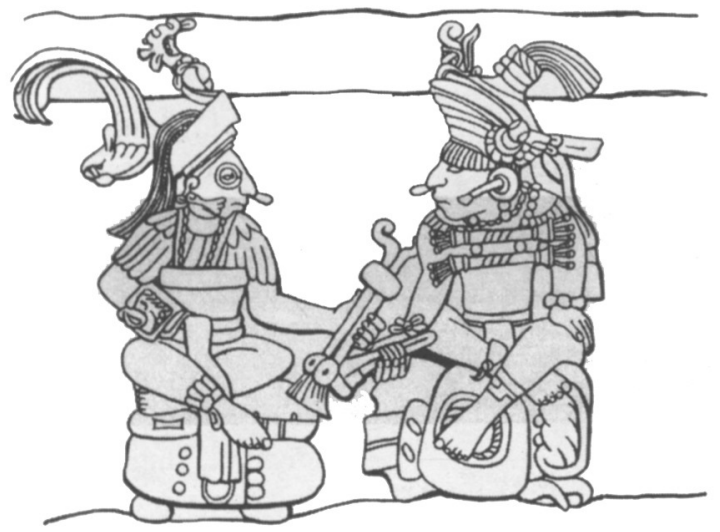

Fig. 11. Two conference participants discussing the Trapezium Cluster during a coffee break.

It's difficult for me to remember exactly who was the originator of this idea about our understanding of close binary stars. It may have originated with Otto Struve (when confounded by the complexities of $\beta$ Lyrae). However, I heard the same tale from F.B. Wood, Dan Popper and Mirek Plavec separately over the years. I paraphrase them: 'One of worst possible things that could befall researchers of interacting binary stars would to be taken (after death or by an UFO) to the astronomical objects of their attention (and often affection) and to be confronted by the star systems as they really are'. Now with new techniques, such as long baseline interferometry and direct imaging with $\mathrm{AO}$ techniques, or from space with large telescopes, it may not be long before we will actually "see" what we have previously inferred (or modeled) from the observations. I look forward to this day with some dread, but mostly with great expectations to see and understand the beauty and intricate complexities of the binary and multiple star systems.

I wish to thank Colin Scarfe and Christine Allen for inviting me to participate in this colloquium honoring Arcadio Poveda. I have known Arcadio for over 15 years and it is fitting that this meeting was held in his hometown of Mérida. Also, it is very fitting that it occurs at a time when so much progress is being made in the study of binary and multiple star systems, a field that Arcadio loves and helped shape. Thanks also is given to the local organizing committee, who did an outstanding job in organizing the meeting and providing many superb cultural events and visits. I also wish to thank Larry DeWarf at Villanova University for preparing the text and figures for publication, as well as Scott Engle for helping to proofread the manuscript before publication.

\section{REFERENCES}

Duquennoy, A. \& Mayor, M. 1991, A\&A, 248, 458

Guinan, E. F. 2004, New Astr. Rev., 48, 647

Öpik, E. 1925, AN, 223, 73

Öpik, E. 1929, AN, 235, 265

Taylor, S. F., Harvin, J. A., \& McAlister, H. A. 2003, PASP, 115, 609

Editors' note: As is also indicated in the Preface, the papers referred to in this summary by Yousaf Butt, Thierry Forveille, Edward Guinan, Rosemary Mardling and Bob Mathieu are not included in this volume because no manuscripts were received by the editors. We can forgive Ed Guinan, however, in gratitude for his preparation of this summary.

Edward F. Guinan, Villanova University, 800 E. Lancaster Ave., Villanova, PA 19085, U.S.A. (edward.guinan@ villanova.edu). 